\title{
PERFIL DIAGNÓSTICO DE ENFERMAGEM PARA PESSOAS COM LEISHMANIOSE
}

\section{PROFILE DIAGNOSIS OF NURSING FOR PEOPLE WITH LEISHMANIOSE}

\section{DIAGNÓSTICO PERFIL DE ENFERMERÍA PARA PERSONAS CON LEISHMANIASIS}

Vinicius Lino de Souza Neto ${ }^{1}$, Cristiane da Silva Costa ${ }^{2}$, Isabella Kilia Macedo Silva ${ }^{3}$, Rosângela Vidal de Negreiros ${ }^{4}$, Ellen Caroline Paiva de Godoy ${ }^{3}$, Bárbara Coeli Oliveira da Silva ${ }^{5}$, Richardson Augusto Rosendo da Silva ${ }^{6}$

\begin{abstract}
RESUMO
Objetivo: identificar o perfil diagnóstico de enfermagem para pessoas com Leishmaniose visceral em uma unidade de infectologia. Métodos: estudo transversal, descritivo, com abordagem quantitativa, realizado em 36 pessoas com Leishmaniose Visceral que estavam presentes na unidade de infectologia de um Hospital Universitário, no Nordeste do Brasil. Para a coleta de dados, adotouse um roteiro de anamnese e exame físico. Os diagnósticos foram construídos processualmente utilizando a CIPE versão 2015. Resultados: foram identificados 57 focos da prática de enfermagem e elaborados 35 enunciados, validando-se 13 afirmativas de diagnósticos, sendo estes: frequência cardíaca aumentada, eliminação intestinal excessiva, tremor, medo, defecação comprometida, estado de consciência alterado, aceitação do regime terapêutico prejudicado, hipertermia, socialização, exposição à contaminação, sangramento, insônia e o uso de drogas. Conclusão: por fim, nota-se que o perfil diagnóstico direciona as intervenções prioritárias a pessoas com LV.

Descritores: Processos de enfermagem; Cuidados de enfermagem; Doenças transmissíveis; Leishmaniose visceral.
\end{abstract}

\section{ABSTRACT}

Objective: To identify the profile nursing diagnosis for people with visceral leishmaniasis and infectious diseases unit. Methods: Cross-sectional, descriptive study with a quantitative approach, performed with 36 people with Visceral Leishmaniasis who were present in the infectious diseases unit of a university hospital in northeastern Brazil. For data collection adopted a script history and physical examination. Diagnoses were built procedurally using CIPE version 2015. Results: We identified 57 nursing practice focuses, and prepared 35 statements, if validating 13 affirmative diagnostics, namely: increased heart rate, excessive bowel movements, tremor, fear, committed defecation, altered state of consciousness, acceptance of impaired therapeutic regimen, hyperthermia, socialization, exposure to infection, bleeding, insomnia and the use of drogas. Conclusion: finally, note that the diagnostic profile directs the priority interventions will people LV.

Descriptors: Nursing process; Nursing care; Communicable diseases; Leishmaniasis visceral.

\section{RESUMEN}

Objetivo: Identificar los diagnósticos de enfermería perfil de las personas con leishmaniasis visceral y la unidad de enfermedades infecciosas. Métodos: transversal, descriptivo, con abordaje cuantitativo, realizado con 36 personas con leishmaniasis visceral que estaban presentes en la unidad de enfermedades infecciosas de un hospital universitario en el noreste de Brasil. Para la recolección de datos adoptado una historia de la escritura y la exploración física. Los diagnósticos fueron construidos usando procesalmente CIPE versión de 2015. Resultados: Se identificaron 57 la práctica de enfermería se centra, y se prepararon 35 estados, si la validación de 13 diagnósticos afirmativas, a saber: aumento del ritmo cardíaco, movimientos excesivos del intestino, temblor, miedo, defecación comprometida, estado alterado de conciencia, de aceptación del sistema alteración de la hipertermia terapéutica, la socialización, la exposición a la infección, hemorragia, el insomnio y el consumo de drogas. Conclusión: Por último, cabe destacar que el perfil de diagnóstico dirige las intervenciones prioritarias las personas con LV.

Descriptores: Procesos de enfermería; Atención de enfermería; Enfermedades transmisibles; Leishmaniasis visceral.

${ }^{1}$ Graduado em Enfermagem. Mestre em Enfermagem. Professor Substituto do Curso de Enfermagem da Universidade Federal do Rio Grande do Norte. ${ }^{2}$ Graduada em Enfermagem pela Universidade Federal do Rio Grande do Norte. ${ }^{3}$ Graduanda do Curso de Enfermagem da Universidade Federal do Rio Grande do Norte. ${ }^{4}$ Graduada em Enfermagem. Mestra em Enfermagem. Professora do Curso de Enfermagem da Universidade Federal de Campina Grande. ${ }^{5}$ Graduada em Enfermagem. Doutoranda em Enfermagem. Professora Substituta do Curso de Enfermagem da Universidade Federal do Rio Grande do Norte. ${ }^{6}$ Graduado em Enfermagem. Doutor em Ciências da Saúde. Professor do Curso de Enfermagem e do Programa de Pós-Graduação em Enfermagem da Universidade Federal do Rio Grande do Norte. 


\section{INTRODUÇÃO}

A leishmaniose visceral (LV) é uma das principais doenças tropicais sistemáticas, negligenciadas e fatais, compreendendo uma taxa anual de incidência de 202.000 - 389.000 casos registrados e cerca de 20.000 - 40.000 mortes no mundo ${ }^{(1)}$.

Atualmente, a LV é endêmica em 62 países, com um total estimado de 200 milhões de pessoas sob o risco de adquirirem a infecção. Os maiores índices de casos ocorrem nos países como Índia, Bangladesh, Nepal, Sudão e Brasil, correspondendo a uma taxa de $90 \%^{(1-2)}$. No Brasil, a LV tem maior incidência em áreas rurais, pois dentre os 27 estados a média nos últimos cinco anos foi de 3.500 casos novos $^{(3)}$.

A sintomatologia da doença é caracterizada por febre de longa duração, perda de peso, astenia, adinamia e anemia. Assim, o cuidado implicado pelos profissionais de saúde no âmbito multiprofissional requer habilidade e competências embasadas a partir de evidências científicas e o uso de tecnologias, sejam leve, leve-dura ou dura ${ }^{(4)}$.

Diante disso, o Processo de Cuidar de Enfermagem, realizado por uma equipe, supervisionado pelo enfermeiro, deve implementar ações e intervenções sistemáticas direcionando-se às necessidades prioritárias do paciente e otimizando tempo que reflete na qualidade de vida da pessoa com $\mathrm{LV}^{(5)}$.

Assim, a Sistematização da Assistência de Enfermagem (SAE) é um método utilizado pelo enfermeiro que proporciona ganhos e desafios, pois requer do profissional a prática do pensamento crítico e a utilização do raciocínio clínico, diagnóstico e terapêutico. Dentre as diversas maneiras de se implementar a SAE, pode-se utilizar os modelos de decisões, Processo de Enfermagem (PE), o plano de cuidados, entre outros $^{(6)}$.

O PE é um método que caracteriza-se pela sua praticidade, otimização, com relevância científica, composto por fases inter-relacionadas, interdependentes e recorrentes, como histórico, diagnóstico, intervenções e implementação ${ }^{(7)}$.

Observando a necessidade de complementar o processo, o Conselho Internacional de Enfermeiras (CIE) elabora a Classificação Internacional para a Prática de Enfermagem - CIPE ${ }^{\circ}$, que é considerado como um sistema unificador de linguagem de enfermagem, que permite projetar tendências sobre as necessidades dos pacientes, a provisão de tratamentos, bem como a utilização de recursos e resultados dos cuidados de enfermagem ${ }^{(8)}$.

Assim sendo, a justificativa do estudo pauta-se em identificar as necessidades prioritárias das pessoas com LV para que assim seja possível elaborar uma assistência baseada nos preceitos da evidência científica e implementar ações e intervenções de enfermagem de forma sistemática, acolhedora e humanizada.

Nesse sentido, emergiu-se o seguinte questionamento: Será que a partir dos focos da prática de enfermagem é possível elaborar os diagnósticos de enfermagem para pacientes com LV? Assim, o estudo teve como objetivo identificar o perfil diagnóstico de enfermagem para pessoas com Leishmaniose visceral.

\section{MÉTODO}

Trata-se de um estudo transversal, descritivo, com abordagem quantitativa, realizado na unidade de infectologia de um Hospital Universitário, no Nordeste do Brasil. A população do estudo constituiu-se de todos os sujeitos que estavam presentes no momento da coleta. Para calcular a amostra, adotou-se por base a média aritmética de atendimento da unidade para pessoas com leishmaniose entre 2010 e 2014 que foi de 126 por ano. A fórmula utilizada foi para populações finitas levando em consideração o nível de confiança de $95 \%$ e o tamanho da população $(\mathrm{N}=126)^{(9)}$.

A seleção das 36 pessoas com LV foi por conveniência de forma consecutiva, adotando-se os seguintes critérios de inclusão: ter sido diagnosticado clinicamente com Leishmaniose visceral, apresentar idade acima de 18 anos, estar internado no hospital no período da coleta de dados. Utilizou-se como critérios de exclusão: desconhecer o diagnóstico da doença e não estar em condições psíquicas e emocionais.

A fim de verificar a condição psíquica dos pacientes com Leishmaniose, os pesquisadores previamente se reportavam ao prontuário para analisar todo o histórico da doença e a evolução. Em seguida, reportavam-se à equipe de 
enfermagem para obter informações adicionais sobre o comportamento do paciente, sua orientação quanto ao espaço e tempo.

Os dados foram coletados no período de fevereiro a outubro de 2014, por meio de um roteiro de anamnese e exame físico que contemplou os aspectos sociodemográficos, clínicos e comportamentais ${ }^{(10)}$. O presente instrumento foi submetido à validação do conteúdo e aparência por dez docentes que desenvolvem estudos na área da SAE; posteriormente, as sugestões propostas foram contempladas no instrumento.

Em seguida, foi realizado um treinamento teórico e prático para padronizar a coleta de dados com dois alunos de Iniciação Científica e três alunos de pós-graduação a nível de Mestrado com carga horária de 12 horas semanais, desenvolvido por meio de aulas expositivas e dialogadas e discussões de casos clínicos com ênfase na abordagem a pacientes com Leishmaniose.

Após a etapa teórica do curso, realizou-se uma atividade prática de simulação de exame físico em pares com o intuito de capacitar os pesquisadores e uniformizar a coleta de dados. Assim, após essa etapa, foi realizada sob a forma de pré-teste a aplicação do instrumento em $10 \%$ da amostra.

A elaboração dos diagnósticos foi processual, realizada simultaneamente com a coleta de dados, buscando identificar os focos da prática de enfermagem para que fossem construídos os enunciados de diagnóstico. Assim, foi utilizada a CIPE $^{\oplus}$ versão 2015, que é um sistema de informação com o objetivo de complementar na elaboração do diagnóstico. Segundo o Conselho Internacional de Enfermagem (ICN), para elaborar um diagnóstico de enfermagem, o enfermeiro deve utilizar um termo do eixo foco e julgamento ${ }^{(11)}$.

Após a construção, as afirmativas diagnósticas de enfermagem foram submetidas a um processo de validação de conteúdo. Assim, foi elaborada uma planilha no Microsoft Excel versão 2009 com as respectivas afirmativas de diagnósticos de enfermagem para pacientes com Leishmaniose.

Com isso, foi solicitada a colaboração de três enfermeiros assistenciais e de dois docentes de enfermagem que atuavam na clínica. Tais profissionais foram incluídos na validação, tendo em vista sua experiência e especialidade na clínica. Sua incumbência foi avaliar se as afirmativas propostas eram aplicáveis a pacientes com leishmaniose. Em caso de discordância das afirmativas, requisitou-se que fossem apresentadas sugestões para sua adequação à realidade da prática de enfermagem.

O tratamento dos dados foi realizado por meio da estatística descritiva que elaborou o banco utilizando o Microsoft Excel 2009 cujos resultados foram apresentados descritivamente sob a forma de tabelas por meio de distribuição de frequência (f) e percentual (\%). Logo em seguida, para análise do grau de concordância entre o pesquisador e os especialistas, optou-se pelo índice de Kappa, analisado pelo programa Statistical Package for the Social Science (SPSS), versão 22.0 .

O índice de Kappa foi definido como uma medida de associação para descrever e testar o grau de concordância, ou seja, confiabilidade e precisão de uma avaliação. Valores de Kappa $\geq$ 0.80 são considerados como um bom nível de concordância. Para fins de validação dos diagnósticos de Enfermagem, foram considerados apenas os que tivessem concordância substancial de 0,61 a 0,80 e excelente acima de 0,80.

Assim, o estudo atendeu aos preceitos éticos de pesquisa envolvendo seres humanos segundo normas nacionais e internacionais com parecer favorável do Comitê de Ética em Pesquisa do Hospital Universitário, Brasil, parecer $\mathrm{n}$ 은 510.708. Antes de iniciar a coleta de dados, os participantes do estudo foram esclarecidos acerca do objetivo da pesquisa, e foi realizada uma leitura do Termo de Consentimento Livre e Esclarecido (TCLE).

\section{RESULTADOS E DISCUSSÃO}

Participaram do estudo 36 pessoas com Leishmaniose Visceral, com idade mínima de 35 anos e máxima de 45 anos (67,75\%). Na maioria eram mulheres $(57,78 \%)$, casadas $(63,85 \%)$, autônomas (41,33\%), caucasianas (54,21\%) e católicas $(85,74 \%)$. Nesse sentido foram identificados 57 focos da prática de enfermagem e elaborados 35 enunciados, validando-se 13 afirmativas de diagnósticos. 
Tabela 1- Distribuição de Diagnósticos de Enfermagem para pessoas com Leishmaniose, Natal -RN, Brasil. (2014).

\begin{tabular}{lll}
\hline Diagnóstico de Enfermagem & IC > 0,80 & $\mathbf{f ( \% )}$ \\
Eliminação intestinal excessiva & 1,0 & 89 \\
Defecação comprometida & 0,80 & 88 \\
Frequência cardíaca aumentada & 1,0 & 84 \\
Tremor & 1,0 & 81 \\
Hipertermia & 1,0 & 75 \\
Sangramento & 0,90 & 71 \\
Estado de consciência alterado & 0,90 & 70 \\
Medo & 1,0 & 69 \\
Insônia & 0,82 & 63 \\
Aceitação do regime terapêutico prejudicado & 0,80 & 61 \\
Uso de drogas & 0,80 & 60 \\
Socialização & 0,90 & 59 \\
Exposição à contaminação & 0,80 & 58 \\
\end{tabular}

Fonte: Dados da pesquisa, 2014. Nota: IC - Índice de Concordância.

Os cuidados implicados às pessoas com Leishmaniose visceral (LV) requerem do enfermeiro uma habilidade e competência capaz de identificar as necessidades prioritárias. O perfil diagnóstico surge como uma ferramenta que tem como objetivo direcionar as metas de cuidados das pessoas com leishmaniose por meio das intervenções de enfermagem ${ }^{(12)}$.

Assim, neste estudo, por ter sido encontrado um grande quantitativo de diagnósticos de enfermagem, priorizou-se pelas afirmativas que apresentaram índice de concordância maior ou igual a 1.0, sendo estes: frequência cardíaca aumentada, eliminação intestinal excessiva, tremor, medo e hipertermia.

O diagnóstico de eliminação intestinal excessiva foi conceituado a partir do aumento do número de evacuações realizadas ao dia e de aspecto aquoso ${ }^{(11)}$. Em pacientes com Leishmaniose, a causa do quadro está vinculada ao agente etiológico da doença e à terapêutica implicada. Os fármacos utilizados no tratamento possuem como efeito adverso a instabilidade da pressão oncótica e hidrostática no lúmem intestinal, levando ao quadro de diarreia ${ }^{(13)}$.

Diante desse panorama o enfermeiro deve implementar intervenções que atenuem ou melhorem as condições fisiológicas do paciente, como: mensuração do Índice de Massa Corpórea (IMC), solicitação de avaliação nutricional, orientação quanto aos hábitos alimentares saudáveis, passagem de sonda nasogástrica em casos de necessidade, cuidados específicos com as sondas (identificação da funcionalidade, controle no tempo de permanência e higienização), avaliação da suplementação na alimentação do paciente junto com o serviço de nutrição, hidratação oral/venosa, balanço hídrico e monitorização do estado hidroeletrolítico ${ }^{(13)}$.

O diagnóstico de frequência cardíaca aumentada caracteriza-se pelo aumento do volume sistólico ${ }^{(11)}$. A causa para o desenvolvimento do quadro ainda não é bem elucidada, todavia o enfermeiro deve estar atento para tal necessidade, pois o quadro afeta diretamente os demais sistemas do organismo, aumentando o risco de doenças cardiovasculares $^{(13)}$.

Dentro das várias formas de vigilância e intervenção de enfermagem, as ações prioritárias incluem a ausculta cardíaca conforme a necessidade, mensuração dos sinais vitais, administração de medicamentos, monitorização cardíaca e cuidados relacionados à alimentação hipersódica ${ }^{(13)}$.

Outra manifestação observada nas pessoas com LV foi o desenvolvimento do quadro de tremor e hipertermia. De acordo com a CIPE $^{\circ}$ versão 2015, o tremor é caracterizado como contrações musculares involuntárias alternadas com o relaxamento muscular ${ }^{(11)}$. Essa manifestação é evidenciada pelo aumento 
considerável do nível de toxinas liberadas pelo agente etiológico ${ }^{(14)}$.

No entanto, o tremor pode colocar o paciente em risco, deixando-o suscetível a quedas, e caso tenha como gravidade à perda de proteína muscular, muitos podem ficar restritos ao leito, vindo assim a desenvolver enfermidades secundárias, por exemplo, a úlcera por pressão ${ }^{(15-12)}$.

Em relação ao diagnóstico de hipertermia, este caracteriza-se pela diminuição da capacidade em regular o termostato interno, pois o agente etiológico afeta os hepatócitos, danificando as células e liberando termotoxinas ${ }^{(11)}$. Para tentar diminuir a recorrência do quadro, o enfermeiro deve realizar intervenções, como aprazar e administrar as medicações de horário, fazer compressa em locais de grande circulação, prescrever mudança de decúbito e orientar quanto ao uso de roupas leves ${ }^{(16-17)}$.

O medo foi outro diagnóstico frequente nas pessoas com LV. Tal sentimento é referido a um anseio relacionado à emoção decorrente de uma causa real e associada ao perigo, tendo função de defesa, pois gera uma reação no organismo ao perigo iminente ${ }^{(11)}$. Além disso, o medo desencadeia reações fisiológicas de fuga referentes a situações desafiadoras; dessa forma, o sentimento apresentado influencia diretamente na aceitação do diagnóstico, retardando o início do tratamento medicamentoso ${ }^{(17)}$.

Assim, o enfermeiro deve prestar uma assistência educativa com o objetivo de orientar e esclarecer os cuidados implicados na terapia, bem como proporcionar o apoio individual e coletivo; oferecer conforto ao paciente; orientar e promover atividades em grupos familiares; e estabelecer vínculo de apoio familiar para assim obter uma assistência efetiva e qualificada ${ }^{(15-17)}$.

\section{CONCLUSÃO}

Após a análise dos dados, delineou-se o perfil diagnóstico de enfermagem a pessoas com leishmaniose composto por 57 focos da prática de enfermagem, 35 enunciados, validando-se 13 afirmativas de diagnósticos.

Dentre as diversas limitações do estudo, teve-se a limitação geográfica, pois desenvolveuse em um estado do Nordeste. Porém, acreditase que a evidência científica proporcionada por esse estudo incentive novas pesquisas para complementar e difundir a aplicabilidade da CIPE no âmbito da infectologia.

Desse modo, espera-se que a evidência científica proporcionada pelo estudo possibilite um aprimoramento tanto aos enfermeiros da prática clínica como para aos campos da pesquisa, extensão, ensino e gerência.

\section{REFERÊNCIAS}

1. Alvar J, Velez ID, Bern C, Herrero M, Desjeux P, Cano J, et al. Leishmaniasis worldwide and global estimates of its incidence. PLOS ONE. 2012;7(5):1$12 . \quad$ Disponível em: http://journals.plos.org/plosone/article/asset?id= 10.1371\%2Fjournal.pone.0035671.PDF

2. Dias AALS, Milani D, Santos EC, Ornelas J, Figueiredo RM, Yamamura M. Produção nacional e acesso sobre enfermagem e doenças transmissíveis. UNOPAR Cient. Ciênc. Biol. Saúde. 2011;13(esp):299-306. Disponível em: http://www.pgsskroton.com.br/seer/index.php/J HealthSci/article/viewFile/1076/1032

3. Brustonoli FM, Serra JPA, Souza AB, Pereira EML, Machado LBP, Branco NM et al. Aspectos socioeconômicos e conhecimento de familiares de crianças acometidas pela leishmaniose visceral no Mato Grosso do Sul. Ensaio e Ciência 2013;17(3):71-82. Disponível em: http://pgsskroton.com.br/seer/index.php/ensaio eciencia/article/view/2338/2237

4. Horta WA. Processo de enfermagem. Rio Janeiro: Guanabara Koogan; 2011.

5. Bittencourt GKGD, Crossetti MGO. Habilidades de pensamento crítico no processo diagnóstico em enfermagem. Rev. Esc. Enferm. 2013;47(2):341-7. Disponível em: http://www.scielo.br/pdf/reeusp/v47n2/10.pdf

6. Nóbrega MML, Garcia TR. Perspectivas de incorporação da Classificação Internacional para a Prática de Enfermagem (CIPE ${ }^{\circledR}$ ) no Brasil. Rev. Bras. Enferm. 2005;58(2):227-30. Disponível em: http://www.scielo.br/pdf/reben/v58n2/a20.pdf 7. Mattei FD, Toniolo RM, Malucelli A, Cubas MR. Uma visão da produção científica internacional sobre a Classificação Internacional para a Prática de Enfermagem. Rev. Gaúcha. Enferm. 2011; 32(4):823-31. Disponível em: http://www.scielo.br/pdf/rgenf/v32n4/v32n4a25 . $\mathrm{pdf}$

8. Garcia TR, Nóbrega MML. A terminologia CIPE ${ }^{\circledR}$ e a participação do centro $\mathrm{CIPE}^{\circledR}$ brasileiro em seu desenvolvimento e disseminação. Rev. Bras. Enferm. 2013;66(esp):142-50. Disponível em: 
http://www.scielo.br/pdf/reben/v66nspe/v66nsp ea18.pdf

9. Fontelles MJ, Simões MG, Almeida JC, Fontelles RGS. Metodologia da pesquisa: diretrizes para o cálculo do tamanho da amostra. Rev. Paran. Med. 2010;24(2):57-64. Disponível em: http://files.bvs.br/upload/S/0101-

\section{7/2010/v24n2/a2125.pdf}

10. Andrade LL. Construção de instrumentos para a documentação do processo de enfermagem em uma clínica de doenças infectocontagiosas. João Pessoa. Dissertação [Mestrado em Enfermagem] Universidade Federal da Paraíba; 2012.

11. Catálogo $\mathrm{CIPE}^{\circledR}$ : Enfermagem Comunitária. Ordem dos Enfermeiros; 2015. Disponível em: http://www.ordemenfermeiros.pt/publicacoes/D ocuments/CatalogoCIPE_web_julho2015.pdf.

12. Silva Junior GB, Daher EF. Tropical diseasesassociated kidney injury. Rev. Bras. Clin. Med. 2013;11(2):155-64. Disponível em: http://files.bvs.br/upload/S/16791010/2013/v11n2/a3570.pdf

13. Nettina SM. Prática de enfermagem. 9. ed. Rio de Janeiro: Guanabara Koogan; 2009.

14. Ducan BB, Chor D, Aquino EML, Bensenor IM, Mill JG, Schmidt MI, et al. Doenças crônicas não transmissíveis no Brasil: prioridade para enfrentamento e investigação. Rev. Saúde Pública. 2012; 46(1):126-34. Disponível em: http://www.scielo.br/pdf/rsp/v46s1/17.pdf

15. Luzia MF, Victor MAG, Lucena AF. Nursing Diagnosis Risk for falls: prevalence and clinical profile of hospitalized patients. Rev. Latino-Am. Enfermagem. 2014;22(2):262-8. Disponível em: http://www.scielo.br/pdf/rlae/v22n2/0104-1169rlae-22-02-00262.pdf

16. Costa DL,Rocha RL,Carvalho RM,Lima-Neto $\mathrm{AS}$, Harhay $\mathrm{MO}$,Costa $\mathrm{CH}$, et.al. Serum cytokines associated with severity and complications of kala-azar. Pathog. Glob. Health. 2013;07(2):78-87. Disponível em: http://www.ncbi.nlm.nih.gov/pmc/articles/PMC4 001482/pdf/pgh-107-02-078.pdf

17. Souza Neto VL, Andrade LL, Agra G, Costa MML, Silva RAR. Perfil diagnóstico de enfermagem de pacientes hospitalizados em unidade de infectologia. Rev Gaúcha Enferm. 2015;36(3):79-85. Disponível em: http://seer.ufrgs.br/index.php/RevistaGauchadeE nfermagem/article/view/51495/35059
Nota: Trabalho de pesquisa proveniente de um macro projeto desenvolvido por pesquisadores da UFCG e UFRN.

Recebido em: 19/04/2016

Versão final apresentada em: 30/05/2017

Aprovado em: 05/06/2017

Endereço de correspondência:

Vinicius Lino de Souza Neto

Universidade Federal do Rio Grande do Norte, Centro de Ciências da Saúde - Departamento de Enfermagem Campus Central, s/n, Lagoa Nova CEP: 59078-970 Natal/RN - Brasil

E- mail: vinolino@hotmail.com 